Stage II NEC $(p=0.82)$ or sepsis $(p=0.21)$ nor of stool alpha1 -antitrypsin in those with any NEC $(p=0.70)$; $\geq$ Bells Stage II NEC $(p=0.87)$ or sepsis $(p=0.81)$.

Discussion In this cohort, the SAT by lactulose:mannitol ratios and stool A1AT did not show evidence of increased intestinal permeability at 2 weeks of age in infants who subsequently developed NEC or sepsis.

\section{PS-185 INTESTINAL OXYGEN EXTRACTION STRONGLY CORRELATES WITH I-FABP LEVELS, A MARKER FOR INTESTINAL DAMAGE}

${ }^{1}$ TE Schat, ${ }^{2} \mathrm{FH}$ Heida, ${ }^{2} \mathrm{M}$ Schurink, ${ }^{1} \mathrm{CV}$ Hulzebos, ${ }^{1}$ AF Bos, ${ }^{1} \mathrm{EMW}$ Kooi, ${ }^{2} \mathrm{JBF}$ Hulscher. ${ }^{1}$ Division of Neonatology, Beatrix Children's Hospital/University Medical Center Groningen, Groningen, Netherlands; 'Division of Pediatric Surgery, Department of Surgery/University Medical Center Groningen, Groningen, Netherlands

\subsection{6/archdischild-2014-307384.482}

Background and aim It remains unknown whether near-infrared spectroscopy (NIRS) can be used to assess intestinal perfusion. Intestinal fatty acid binding proteins in plasma (I-FABPp) and urine $(\mathrm{I}-\mathrm{FABPu})$ are a direct measure of intestinal epithelial cell damage that may occur after intestinal hypoperfusion. We measured splanchnic fractional tissue oxygen extraction (FTOE) and correlated these FTOE values with I-FABP levels in preterm infants in the first $16 \mathrm{~h}$ after onset of necrotizing enterocolitis (NEC).

Methods Preterm infants born between October 2010 and November 2012 were prospectively included when NEC was diagnosed (Bell stage $\geq 2$ ). Regional tissue oxygen saturation of the liver $\left(\mathrm{r}_{\text {liv }} \mathrm{SO} 2\right)$ and infra-umbilical $\left(\mathrm{r}_{\text {int }} \mathrm{SO} 2\right)$ region were measured continuously by NIRS. Mean 8-hour FTOE values were calculated: FTOE $=\left(\mathrm{SpO}_{2}-\mathrm{rSO}_{2}\right) / \mathrm{SpO}_{2}$. Plasma and urine samples collected in the first $16 \mathrm{~h}$ after onset of symptoms were

Abstract PS-185 Table 1 Correlation coefficients between FTOE and I-FABP

\begin{tabular}{|ll|l|}
\hline 0-8 hours & & \\
\hline & I-FABPp & I-FABPu \\
\hline livFTOE & $\rho=0.600$ & $\rho=0.500$ \\
& $P=0.285$ & $P=0.667$ \\
& $\mathrm{n}=5$ & $\mathrm{n}=3$ \\
\hline intFTOE & $\rho=0.900$ & $\rho=1.000$ \\
& $P=0.037$ & $P=<0.001$ \\
& $\mathrm{n}=5$ & $\mathrm{n}=3$ \\
\hline 8-16 hours & & \\
\hline & I-FABPp & I-FABPu \\
\hline livFTOE & $\rho=0.881$ & $\rho=0.679$ \\
& $P=0.004$ & $P=0.094$ \\
& $\mathrm{n}=8$ & $\mathrm{n}=7$ \\
\hline intFTOE & $\rho=0.745$ & $\rho=0.833$ \\
& $P=0.013$ & $P=0.005$ \\
& $\mathrm{n}=10$ & $\mathrm{n}=9$ \\
\hline
\end{tabular}

used for analysis. Spearman's correlation test was used to calculate correlation coefficients.

Results Twenty-one preterm infants were included (median [range] gestational age 28 [25-36] weeks, birth weight 1290 [740-2400] grams). Median [range] liver FTOE (livFTOE) was 0.33 [0.07-0.81], infra-umbilical FTOE (intFTOE) 0.48 [0.13$0.82]$, I-FABPp $16.3[0.54-3748] \mathrm{ng} / \mathrm{mL}$, and I-FABPu 89.9 [3.2-23,336] ng/mL. Table 1 shows strong positive correlations between FTOE and I-FABP levels.

Conclusion High intFTOE values, suggestive of an impaired intestinal blood flow, correlated strongly with I-FABP, i.e. with the extent of intestinal epithelial cell damage. These results indicate that intestinal NIRS monitoring can be used to assess intestinal perfusion in preterm infants with an impaired intestinal blood flow such as occurs in NEC.

\section{PS-186 BLOOD LACTATE AS A PREDICTIVE MARKER FOR NEONATAL NECROTISING ENTEROCOLITIS (NEC) SEVERITY AND OPERATIVE OUTCOMES}

AK Davenport, S Chuang, P Desai. Neonatal Unit, Chelsea and Westminster NHS Foundation Trust, London, UK

\subsection{6/archdischild-2014-307384.483}

Background and aims Lactate as a marker for tissue perfusion and hypoxia is increasingly used in routine point-of-care monitoring in critical care. We investigated the association of preoperative lactate (PreL) with operative outcomes and mortality in neonatal surgical NEC.

Methods 25 infants with NEC confirmed on laparotomy at a tertiary surgical centre were retrospectively evaluated. Maximal PreL was categorised as normal $(<2 \mathrm{mmol} / \mathrm{L})$, moderate $(2-5$ $\mathrm{mmol} / \mathrm{L})$ or severe $(>5 \mathrm{mmol} / \mathrm{L})$, and correlated to extent of NEC involvement and mortality.

Results Median birth gestation and weight were 27weeks (range 23-33) and 1035g (555-2060). Median PreL was 5.1(range 0.616.2) $\mathrm{mmol} / \mathrm{L}$. Elevated PreL correlated with NEC severity, with predominance of pan-intestinal and multifocal involvement in severe hyperlactaemia (Table 1). Infants with isolated NEC were observed to have normal or moderate-PreL. Moderate to severe PreL was also associated with increased mortality rate and need for further surgery.

Conclusions This preliminary study suggests that pre-operative hyperlactaemia and degree of elevation may be associated with a poor prognosis in infants with surgical NEC. Further larger studies may enable better evaluation of its use an adjunctive monitoring or prognostic tool in guiding early neonatal NEC management.

Abstract PS-186 Table 1 Surgical outcomes and Preoperative lactate (PreL)

\begin{tabular}{llll}
\hline & $\begin{array}{l}\text { Normal-PreL } \\
(<2 \mathrm{mmol} / \mathrm{L}) \\
(\mathrm{n}=3)\end{array}$ & $\begin{array}{l}\text { Moderate-PreL } \\
(2-5 \mathrm{mmol} / \mathrm{L})\end{array}$ & $\begin{array}{l}\text { Severe-PreL } \\
(\mathrm{n}=5 \mathrm{mmolL})\end{array}$ \\
\hline Pan-NEC $(\mathrm{n}=13)$ & $0(0 \%)$ & $3(33.3 \%)$ & $10(76.9 \%)$ \\
Multifocal-NEC $(\mathrm{n}=6)$ & $1(33.3 \%)$ & $2(22.2 \%)$ & $3(23.1 \%)$ \\
Isolated-NEC $(\mathrm{n}=6)$ & $2(66.7 \%)$ & $4(44.4 \%)$ & $0(0 \%)$ \\
Further surgery* $(\mathrm{n}=7)$ & $0(0 \%)$ & $2(22.2 \%)$ & $5(38.5 \%)$ \\
Mortality $(\mathrm{n}=6)$ & $0(0 \%)$ & $2(22.2 \%)$ & $4(30.8 \%)$ \\
\hline
\end{tabular}

